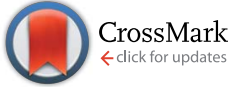

Cite this: Chem. Sci., 2017, 8, 621

Received 14th July 2016

Accepted 25th August 2016

DOI: $10.1039 / c 6 s c 03109 a$

www.rsc.org/chemicalscience

\title{
Reagent-controlled enantioselectivity switch for the asymmetric fluorination of $\beta$-ketocarbonyls by chiral primary amine catalysis $\uparrow$
}

\author{
Yang'en You, ${ }^{\text {ab }}$ Long Zhang ${ }^{\text {abc }}$ and Sanzhong Luo*abc \\ A reagent-controlled enantioselectivity switch was uncovered in the asymmetric $\alpha$-fluorination of $\beta$ - \\ ketocarbonyls by a chiral primary amine catalyst. By a simple swap of fluorination reagents, both \\ enantiomers of the quaternary fluorination adducts could be obtained with good yields and high \\ enantioselectivity. Mechanistic studies disclosed dual $\mathrm{H}$-bonding and electrostatic stereocontrolling \\ modes for the catalysis.
}

Asymmetric catalysis is arguably the most effective and atomeconomic approach for access to optically pure compounds. Using a single chiral catalyst to get two enantiomeric products is attractive as it eliminates the need for the synthesis of catalyst enantiomers, which is not a trivial effort in most cases. ${ }^{1}$ Recently, a switch of enantioselectivity has been noted in a number of metal catalyzed reactions by simply varying the solvent, temperature and additives without structural modifications of the chiral ligands. ${ }^{2}$ However, such an external switch with good enantioselectivity for both enantiomers is still very rare for organocatalytic reactions. ${ }^{3}$ Nagasawa successfully achieved a solvent-switch of enantioselection in an asymmetric Mannich reaction with middle to high ee. Maruoka developed asymmetric aldol and Mannich reactions by an achiral-acidadditive induced switch. Matsubara reported a procedurecontrolled enantioselectivity switch with moderate ee. Herein, we reported an enantioselective switch for synthetically important asymmetric fluorination reactions by two different stereocontrolling modes.

Catalytic enantioselective construction of carbon-fluorine bonds is of significant synthetic interest due to the prevalence of fluorinated drugs and agricultural agents. ${ }^{4 d, h}$ In this regard, enamine catalysis has appeared as a prominent strategy for the fluorination of aldehydes and ketones. Pioneering works by the groups $^{7}$ of Jørgensen, Barbas and MacMillan have achieved asymmetric $\alpha$-fluorination of linear aldehydes with high

\footnotetext{
aBeijing National Laboratory for Molecule Sciences (BNLMS), Key Laboratory for Molecular Recognition and Function, Institute of Chemistry, The Chinese Academy of Sciences, Beijing 100190, China. E-mail: luosz@iccas.ac.cn

${ }^{b}$ University of Chinese Academy of Sciences, Beijing, 100049, China

${ }^{c}$ Collaborative Innovation Center of Chemical Science and Engineering, Tianjin, 300071, China

$\dagger$ Electronic supplementary information (ESI) available: Experimental details including characterization date, copies of ${ }^{1} \mathrm{H},{ }^{13} \mathrm{C}$, and ${ }^{19} \mathrm{~F}$ NMR and HPLC traces. See DOI: 10.1039/c6sc03109a
}

enantioselectivity. The reaction has recently been extended to $\alpha$ branched aldehydes by primary amine catalysis. ${ }^{8}$ In contrast, the asymmetric fluorination of ketones by amine catalysis has remained far less developed, particularly for branched ketones. Two elegant contributions along this line have been recently reported for the reactions of cyclic ketones by the research groups of MacMillan ${ }^{9}$ and Toste. ${ }^{10}$ However, the asymmetric $\alpha$ fluorination of acyclic ketones remains largely undeveloped not only in aminocatalysis, but in general in asymmetric catalysis. Previously, Lewis acid catalysis (Scheme 1, I) has been frequently attempted in the asymmetric fluorination of acyclic ketones, ${ }^{4-6}$ but successes have only been seen with $\beta$-ketoesters bearing bulky (e.g. $t$-butyl) ester groups. ${ }^{5}$ We have pursued the fluorination reaction of acyclic $\beta$-ketocarbonyls using our primary amine catalysts ${ }^{12}$ and during this process we observed an unprecedented reagent controlled switch of enantioselectivity. Thus, by simply choosing two readily available fluorination reagents, one single chiral primary amine catalyst would be able to provide two enantiomers, respectively, bearing fluorinated quaternary centers with good enantioselectivity in both cases (Scheme 1, II).

Our initial investigation was performed with acetoacetate 1a in the presence of our typical primary amine catalyst $\mathbf{I} / \mathrm{TfOH}$. A quick survey of different fluorination reagents was conducted. The reaction proceeded smoothly in all the tests and a switch of chiral induction was clearly noted among different fluorination reagents. While the reaction with NFSI (2a) gave 51\% ee (Table 1 , entry 1 ), the use of $\mathbf{2 b}$ and $\mathbf{2 c}$ led to $-71 \%$ ee and $-38 \%$ ee, respectively, with opposite chiral induction (Table 1, entries 2 and 3). A switch of chiral induction was generally observed with other primary-tertiary diamine catalysts such as IV and $\mathbf{V}$ (Table 1, entries 7-10). Though enantioselectivity varied in different solvents (see the ESI for details $\dagger$ ), the switching phenomenon was uniformly observed (Table 1, entries 4-6 vs. 1-3). In all the cases examined, fluorination reagent $2 \mathbf{b}$ showed better 
I. Lewis-acid-catalyzed asymmetric fluorination of acyclic $\beta$-ketoesters ${ }^{5}$

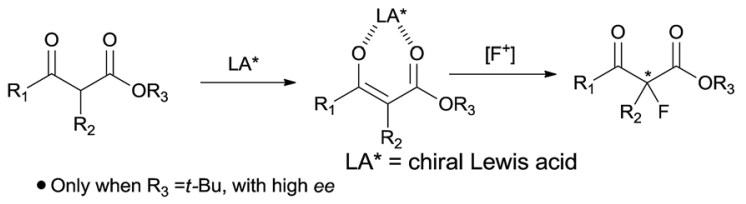

II. Enamine catalysis: Asymmetric Fluorination of acyclic $\beta$-ketocarbonyls
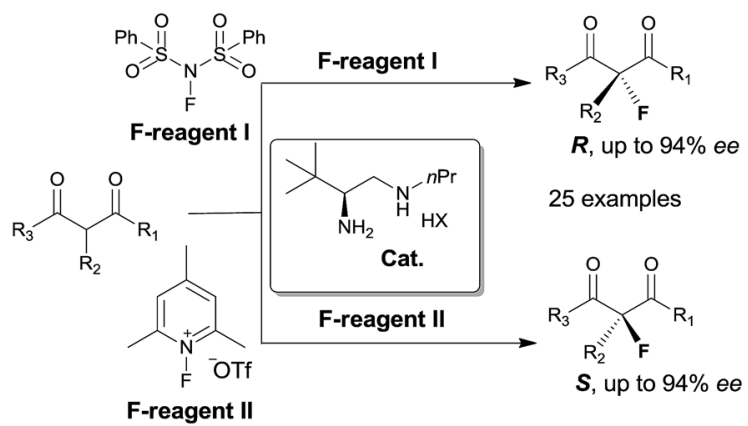

- Broad scopes with $\beta$-ketocarbonyls

- One single catalyst, two enantiomers

- Reagent switching enantioselectivity

Scheme 1 Asymmetric $\alpha$-fluorination of cyclic and acyclic ketones.

enantioselectivity than $\mathbf{2 c}$. The reagents $\mathbf{2 a}$ and $\mathbf{2 b}$ were then selected for subsequent optimization with the aim to find a generally applied enantio-switching catalytic system (see the ESI for details $\uparrow$ ). In this regard, we were delighted to identify a simple primary-secondary diamine II that gave good enantioselectivity in both of the switching reactions (Table 1, entries 11 and 12). Thus, the best reaction conditions are a combination of II/(DNBA I) $(1: 1)$ with $2 \mathbf{a}$ as the fluorination reagent in chloroform at room temperature, providing the product with $86 \%$ yield and $92 \%$ ee, and the configuration of the product is $R$ configuration. On the other hand, when $\mathbf{2 b}$ was used as the fluorination reagent, by the same primary amine II/(DNBA II) in methanol, the desired product was obtained with $95 \%$ yield and $-90 \%$ ee, and the product was $S$-configuration. At this stage, alteration of the acidic additive from $\mathrm{TfOH}$ to dinitrobenzoic acid was found to give a small but noticeable improvement in enantioselectivity (Table 1, entries 13 and $14 v s .11$ and 12). An additional benefit of the use of dinitrobenzoic acid is the ease in handling and manipulation as the resulting salts are crystal solids and bench stable. Adamantyl primary amine III, a close analogue of II, was also found to deliver an excellent $-93 \%$ ee for the $S$-selective process, however, this catalyst performed rather poorly in the $R$-selective process with only $72 \%$ ee (Table 1, entries 15 and 16). This observation indicated a critical balance of steric hindrance for both the two enantio-switching processes.

Under the optimized conditions, the substrate scope for the $R$-selective process was firstly investigated as above by using 20 mol\% II/DNBA I as the catalyst, and $2 \mathbf{a}$ as the fluorination reagent, in $\mathrm{CHCl}_{3}$. As shown in Table 2 , a variety of $\beta$-keto esters, including benzyl, ethyl, isopropyl, allyl, $n$-butyl, cinnamyl, and naphthalen-1-ylmethyl ester all gave the desired products with excellent ee (89-93\% ee) and good yields (up to 99\%) (Table 2, entries 1-7). Alteration of the group $R_{2}, \alpha$-substituents on acetoacetates (3h-3n), was well tolerated. Increasing the bulkiness of the $\alpha$-substituent as in $\mathbf{3} \mathbf{g}-\mathbf{3 k}$ from methyl to iso-butyl led to a reduction in the reactivity, but excellent enantioselectivity was obtained in all these cases (Table 1, entries 8-11). It is noted that the reaction also tolerated $\alpha$-allyl (31), $\alpha$-propargyl (3m) or $\alpha$ benzyl groups (3n), affording the desired fluorination adducts in good yields and high enantioselectivity (Table 2, entries 1214). 1,3-Diketone has been examined in the reaction, showing good reactivity but moderate ee (Table 2 , entry 15), which is likely a result of uncontrolled enol-fluorination due to the predominant enol-form of 1,3-diketones. An ethyl ketone 3p also worked in the reaction, showing low activity and moderate to good enantioselectivity (Table 2, entry 16 ). The observed low reactivity could be ascribed to its difficulty in forming enamine. ${ }^{12}$

The substrate scope could be further extended to $\beta$-ketoamides. ${ }^{11}$ Both $N$-aryl and $N$-aliphatic amides worked well in the reactions with good yields and moderate to good enantioselectivities (Table 2, entries 17-22 and 25). It seems that the free $\mathrm{N}-\mathrm{H}$ moiety has a marginal effect on the reactions. In particular, $N$-aryl amides bearing either electron-donating or electronwithdrawing groups were equally applied, with the latter giving slightly better enantioselectivity (Table 2 , entry 20 vs. 19). A lactone-type substrate $3 \mathbf{w}$ could also be incorporated in the current catalysis with $76 \%$ ee and $99 \%$ yield. ${ }^{6 c}$ Cyclic ketocarbonyls have also been examined, showing good reactivity but moderate enantioselectivity (Table 2, entries 24 and 25).

The same substrate scope was also tested for the $S$-selective reactions. In these cases, the reaction was examined with $2 \mathbf{b}$ as the fluorination reagent, and $20 \mathrm{~mol} \% \mathrm{II} / \mathrm{DNBA}-\mathrm{II}$ or III/HOTf as the catalyst in $\mathrm{CH}_{3} \mathrm{OH}$. In most cases, the reactivity and enantioselectivity were comparable to those obtained in the $R$ selective processes. One particular exception was with the reactions of $\beta$-ketoamides, wherein the $S$-selective process with primary amine catalyst II worked extremely well to afford the desired adducts in good yields and high enantioselectivities (Table 2, entries 17-22 and 25). In comparison, the enantioselectivity with the $R$-process was moderate. The use of primary amine catalyst III/TfOH has been found to deliver improved enantioselectivity in the $S$-selective reactions (Table 2, entries 714 and 23).

To probe the utility of our fluorination reaction in preparative synthesis, a gram-scale reaction of $\beta$-ketoester $1 \mathrm{a}(7.5 \mathrm{mmol}$, $1.545 \mathrm{~g})$ was performed with NFSI $(5 \mathrm{mmol}, 1.576 \mathrm{~g})$ as the fluorination reagent in $\mathrm{CHCl}_{3}$ for $48 \mathrm{~h}$ delivering the desired product 3a with a good yield ( $0.952 \mathrm{~g}, 85 \%$ yield) and excellent enantioselectivity ( $94 \%$ ee). The chiral primary amine catalyst could be quantitatively recovered by concentrating the aqueous solution after work up.

Though an enol-type could not be completely ruled out, the current experimental observations as well as our previous studies strongly favour an enamine mechanism (see the ESI for details $\uparrow$ ). To account for the switch of enantioselectivity, we have proposed two plausible enamine-based intermolecular Fattack transition states. ${ }^{13}$ For the NFSI (2a) based $R$-selective process, an H-bonding mode I between the sulfonyl moiety and 
Table 1 Screening and optimization ${ }^{a}$

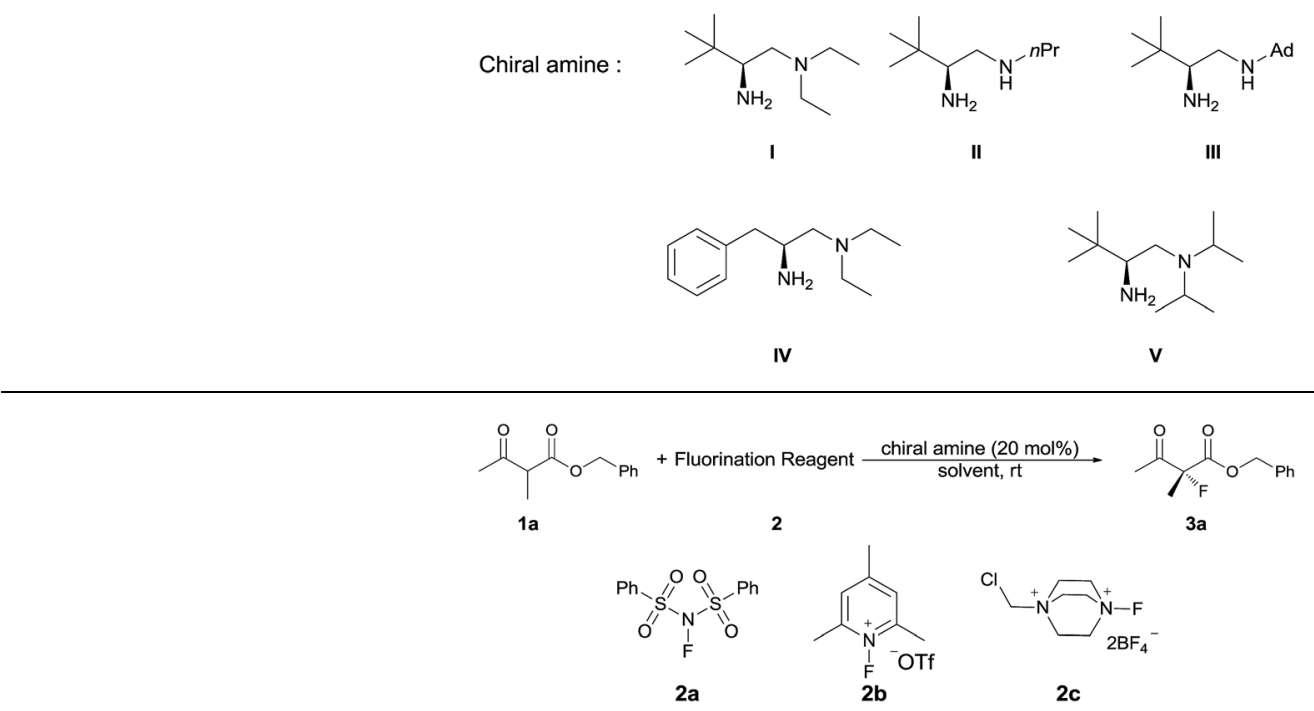

\begin{tabular}{|c|c|c|c|c|c|}
\hline Entry & Amine catalyst & Fluorination reagent & Solvent & Yield $^{b}(\%)$ & $\mathrm{ee}^{c}(\%)$ \\
\hline 1 & I/TfOH & $2 a$ & $\mathrm{CHCl}_{3}$ & 41 & 51 \\
\hline 2 & I/TfOH & $2 \mathbf{b}$ & $\mathrm{CHCl}_{3}$ & 75 & -71 \\
\hline 4 & I/TfOH & $2 a$ & $\mathrm{CH}_{3} \mathrm{OH}$ & 54 & 21 \\
\hline 5 & I/TfOH & $2 \mathbf{b}$ & $\mathrm{CH}_{3} \mathrm{OH}$ & 75 & -83 \\
\hline 6 & I/HOTf & $2 c$ & $\mathrm{CH}_{3} \mathrm{OH}$ & 94 & -69 \\
\hline 9 & V/TfOH & $2 a$ & $\mathrm{CHCl}_{3}$ & 45 & 33 \\
\hline 10 & V/TfOH & $2 \mathbf{b}$ & $\mathrm{CH}_{3} \mathrm{OH}$ & 75 & -93 \\
\hline 11 & II/TfOH & $2 a$ & $\mathrm{CHCl}_{3}$ & 72 & 81 \\
\hline 12 & II/TfOH & $2 \mathbf{b}$ & $\mathrm{CH}_{3} \mathrm{OH}$ & 90 & -89 \\
\hline $13^{d}$ & II/DNBA-I & $2 \mathbf{a}$ & $\mathrm{CHCl}_{3}$ & 85 & 92 \\
\hline $14^{d}$ & II/DNBA-II & $2 \mathbf{b}$ & $\mathrm{CH}_{3} \mathrm{OH}$ & 95 & -90 \\
\hline
\end{tabular}

${ }^{a}$ General conditions: $1 \mathrm{a}(0.075 \mathrm{mmol}), 2(0.05 \mathrm{mmol})$, amine catalyst $(20 \mathrm{~mol} \%)$ in solvent at r.t. for $24 \mathrm{~h}^{b}{ }^{b}$ Isolated yield. ${ }^{c}$ Determined by HPLC on a chiral stationary phase. ${ }^{d}$ DNBA-I: $2,4-\left(\mathrm{NO}_{2}\right)_{2} \mathrm{PhCO}_{2} \mathrm{H}$; DNBA-II: $3,4-\left(\mathrm{NO}_{2}\right)_{2} \mathrm{PhCO}_{2} \mathrm{H}$.

the protonated ammonium $\mathrm{N}-\mathrm{H}$ was invoked in the stereocontrol. In this model, the $\mathrm{Si}$-facial fluorination was largely disfavoured due to geometrically unfavourable H-bonding with the ammonium $\mathrm{N}-\mathrm{H}$. On the other hand, an electrostatic mode II was proposed for the $N$-fluoro-pyridinium (2b) based $S$ selective process, wherein the electrostatic repulsion between the cationic charged ammonium and pyridinium species plays a dominant role. Steric effects would also contribute in this model, however, the impact should be minor, as we note that primary amine II performed equally as well as its more bulky counterparts such as catalysts III and V. The observed solvent effect (e.g. $\mathrm{CH}_{3} \mathrm{OH}$ vs. $\mathrm{CHCl}_{3}$, Table 1, entries 1-6) is also in line with the electrostatic model since ionic species would become highly dissociated in polar protic $\mathrm{CH}_{3} \mathrm{OH}$, a feature favourable for the electrostatic repulsion interaction.

The stereocontrolling modes (I and II) could be further verified by DFT calculations at the B3LYP/6-31G* level of approximation (see the ESI for details $\dagger$ ). For mode II, the $S$ - selective TS- $S$ was favoured over the $R$-selective TS- $R$ by $3.0 \mathrm{kcal}$ $\mathrm{mol}^{-1}$, which is consistent with the experimental observations. We further calculated the electrostatic surface potential (ESP) of the two located TSs. As revealed in Fig. 1b, the ammonium and pyridinium moieties of TS- $R$, both bearing positively charged surfaces, seem not to have any noticeable steric interaction from being close together. Thus, TS- $R$ would be mainly disfavoured by electrostatic repulsion not by steric effects. By AIM analysis, we could also identify an attractive $\mathrm{C}-\mathrm{H} \cdots \mathrm{F}$ interaction between the tert-butyl group of the amine catalyst and the fluorination reagent $\mathbf{2 b}$, as shown in Fig. $\mathbf{1 b}$, which may also contribute in facilitating the $S i$-facial attack in TS-S. ${ }^{\mathbf{1 4}}$

\section{Conclusions}

In summary, we have presented herein a reagent-controlled enantioselectivity switch for the organocatalytic asymmetric fluorination of $\beta$-ketocarbonyls. A simple swap of the 
Table 2 Substrate scope ${ }^{a}$

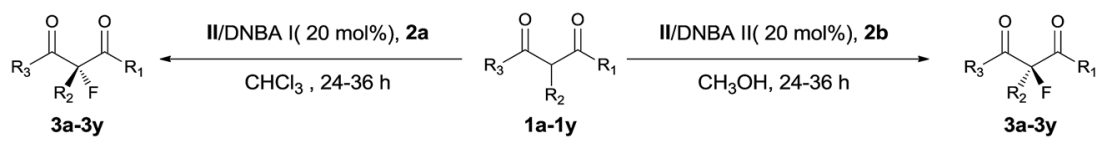

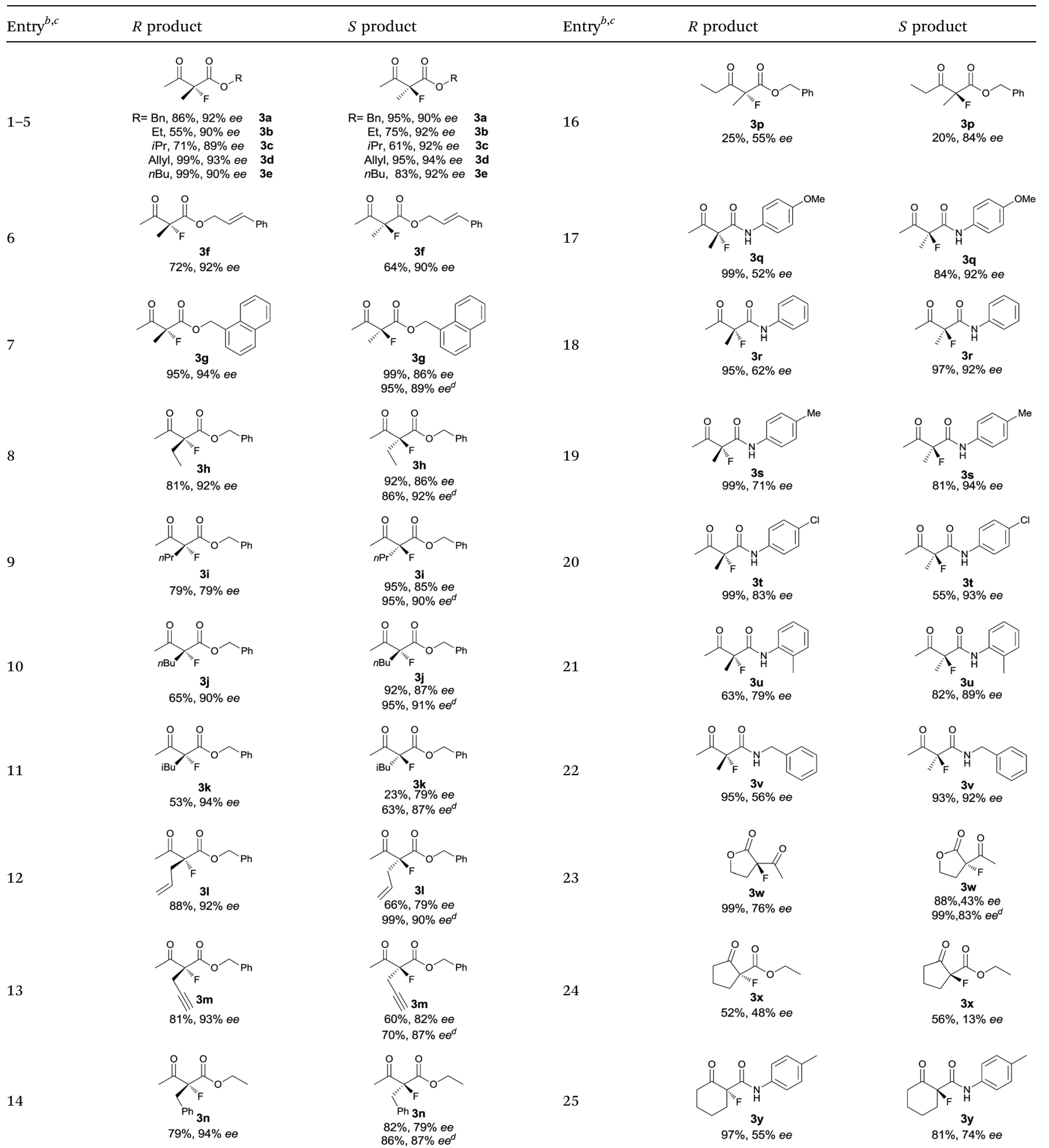


Table 2 (Contd.)

\begin{tabular}{lllll}
\hline Entry $^{b, c}$ & $R$ product & $S$ product & Entry & \\
& & $R$ product & $S$ product \\
\hline
\end{tabular}

15

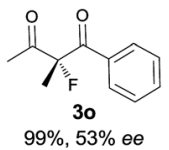

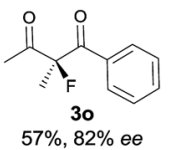

$57 \%, 82 \%$ ee

${ }^{a}$ General conditions: $\mathbf{1}(0.075 \mathrm{mmol}), \mathbf{2 a}(0.05 \mathrm{mmol})$, and II/DNBA-I $(0.01 \mathrm{mmol}, 20 \mathrm{~mol} \%)$ in $\mathrm{CHCl}_{3}(0.25 \mathrm{~mL})$ at r.t. for $24-36 \mathrm{~h} ; \mathbf{1}(0.075 \mathrm{mmol}), 2 \mathbf{b}$ $(0.05 \mathrm{mmol})$, and II/DNBA-II $(0.01 \mathrm{mmol}, 20 \mathrm{~mol} \%)$ in $\mathrm{CH}_{3} \mathrm{OH}(0.4 \mathrm{~mL})$ at r.t. for $24-36 \mathrm{~h} .{ }^{b}$ Yields shown are of isolated products. ${ }^{c}$ The ee was determined by GC or HPLC on a chiral stationary phase. ${ }^{d}$ Catalyst III/TfOH was used instead of II/DNBA-II at r.t. for 36 h.

(a) Proposed transition states I: H-bonding Mode

II: Electrostatic repulsion Mode

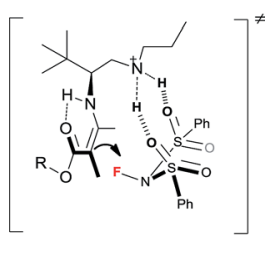

$\boldsymbol{R}$-selective $\mathrm{H}$-bonding guided $R e$-facial attack

(b) Calc

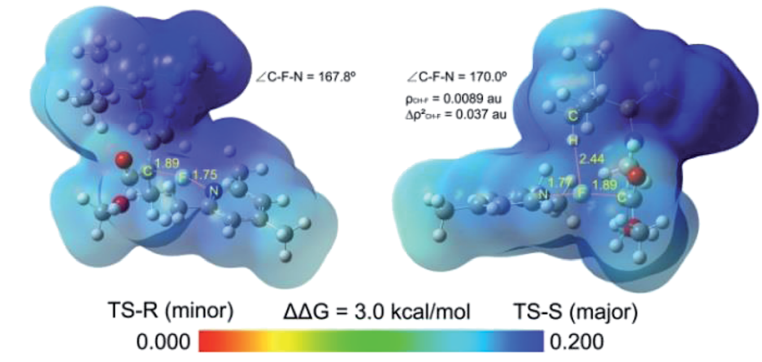

Fig. 1 (a) Proposed transition states (I and II) for the two enantioselectivity switch fluorination reactions; (b) calculated electrostatic surface potential for TSs of the electrostatic mode II.

fluorination reagent switched the enantioselectivity with good reactivity and enantiomeric excess in both cases. Mechanistic studies revealed dual $\mathrm{H}$-bonding and electrostatic stereocontrolling modes for a single chiral primary amine catalyst. Further explorations of switchable enantioselectivity in other reactions are currently underway in our laboratory.

\section{Acknowledgements}

The project was supported by Ministry of Science and Technology (2012CB821600), the Natural Science Foundation of China, and the Chinese Academy of Sciences.

\section{Notes and references}

1 For recent reviews on switching selectivity, see: (a) G. Zanoni, F. Castronovo, M. Franzin, G. Vidari and E. Giannini, Chem. Soc. Rev., 2003, 32, 115; (b) Comprehensive Asymmetric
Catalysis, ed. E. N. Jacobsen, A. Pfaltz and H. Yamamoto, Springer, Berlin, 1999; (c) Catalytic Asymmetric Synthesis, ed. I. Ojima, Wiley-VCH, New York, 2000; (d) V. Blanco, D. A. Leigh and V. Marcos, Chem. Soc. Rev., 2015, 44, 5341; (e) X. Jiang and R. Wang, Chem. Rev., 2013, 113, 5515; (f) T. Tanaka and M. Hayashi, Synthesis, 2008, 3361; $(g)$ M. Bartók, Chem. Rev., 2010, 110, 1663; (h) J. Escorihuela, M. I. Burguete and S. V. Luis, Chem. Soc. Rev., 2013, 42, 5595.

2 For recent examples, see: (a) J. Wang and B. L. Feringa, Science, 2011, 331, 1429; (b) S. Mortezaei, N. R. Catarineu and J. W. Canary, J. Am. Chem. Soc., 2012, 134, 8054; (c) S. A. Moteki, J. Han, S. Arimitsu, M. Akakura, K. Nakayama and K. Maruoka, Angew. Chem., Int. Ed., 2012, 51, 1187; (d) J. Lv, Y. Qin, J. Cheng and S. Luo, Acta Chim. Sin., 2014, 72, 809; (e) Y. Zhang, N. Yang, X. Liu, J. Guo, X. Zhang, L. Lin, C. $\mathrm{Hu}$ and X. Feng, Chem. Commun., 2015, 51, 8432; $(f)$ N. Shibata, T. Ishimaru, T. Nagai, J. Kohno and T. Toru, Synlett, 2004, 10, 1703.

3 For organocatalytic enantioselectivity switches, see: (a) Y. Sohtome, S. Tanaka, K. Takada, T. Yamaguchi and K. Nagasawa, Angew. Chem., Int. Ed., 2010, 49, 9254; (b) A. Garzan, A. Jaganathan, N. Salehi Marzijarani, R. Yousefi, D. C. Whitehead, J. E. Jackson and B. Borhan, Chem.-Eur. J., 2013, 19, 9015; (c) S. A. Moteki, J. Han, S. Arimitsu, M. Akakura, K. Nakayama and K. Maruoka, Angew. Chem., Int. Ed., 2012, 51, 1187; (d) Y. Fukata, K. Asano and S. Matsubara, J. Am. Chem. Soc., 2013, 135, 12160.

4 For recent reviews on asymmetric fluorination reactions, see: (a) P. A. Champagne, J. Desroches, J.-D. Hamel, M. Vandamme and J.-F. Paquin, Chem. Rev., 2015, 115, 9073; (b) T. Furuya, A. S. Kamlet and T. Ritter, Nature, 2011, 473, 470; (c) X. Yang, T. Wu, R. J. Phipps and F. D. Toste, Chem. Rev., 2015, 115, 826; (d) R. Britton and B. Kang, Nat. Prod. Rep., 2013, 30, 227; (e) A. M. R. Smith and K. K. Hii, Chem. Rev., 2011, 111, 1637; (f) S. Lectard, Y. Hamashima and M. Sodeoka, Adv. Synth. Catal., 2010, 352, 2708; $(g)$ J.-H. Lin and J.-C. Xiao, Tetrahedron Lett., 2014, 55, 6147; (h) J. Wang, M. Sánchez-Rosollo, J. L. Acena, C. del Pozo, A. E. Sorochinsky, S. Fustero, V. A. Soloshonok and H. Liu, Chem. Rev., 2014, 114, 2432; (i) V. Bizet, T. Besset, J.-A. Ma and D. Cahard, Curr. Top. Med. Chem., 2014, 14, 901. 
5 For leading examples on metal Lewis acid catalysis, see: (a) L. Hintermann and A. Togni, Angew. Chem., Int. Ed., 2000, 39, 4359; (b) Y. Hamashima, K. Yagi, H. Takano, L. Tamás and M. Sodeoka, J. Am. Chem. Soc., 2002, 124, 14530; (c) N. Shibata, J. Kohno, K. Takai, T. Ishimaru, S. Nakamura, T. Toru and S. Kanemasa, Angew. Chem., Int. Ed., 2005, 44, 4204; (d) A. Narayama, K. Shibatomi, Y. Soga, T. Muto and S. Iwasa, Synlett, 2013, 24, 375.

6 For organocatalytic fluorination of $\beta$-ketocarbonyls, see: $(a)$ D. Y. Kim and E. J. Park, Org. Lett., 2002, 4, 545; (b) X. Wang, Q. Lan, S. Shirakawa and K. Maruoka, Chem. Commun., 2010, 46, 321; (c) J. Xu, Y. Hu, D. Huang, K.-H. Wang, C. Xu and T. Niu, Adv. Synth. Catal., 2012, 354, 515.

7 (a) M. Marigo, D. Fielenbach, A. Braunton, A. Kjærsgaard and K. A. Jørgensen, Angew. Chem., Int. Ed., 2005, 44, 3703; (b) D. D. Steiner, N. Mase and C. F. Barbas, Angew. Chem., Int. Ed., 2005, 44, 3706; (c) T. D. Beeson and D. W. C. MacMillan, J. Am. Chem. Soc., 2005, 127, 8826.

8 (a) S. Brandes, B. Niess, M. Bella, A. Prieto, J. Overgaard and K. A. Jørgensen, Chem.-Eur. J., 2006, 12, 6039; (b) M. R. Witten and E. N. Jacobsen, Org. Lett., 2015, 17, 2772; (c) K. Shibatomi, K. Kitahara, T. Okimi, Y. Abe and S. Iwasa, Chem. Sci., 2016, 7, 1388-1392.

9 (a) P. Kwiatkowski, T. D. Beeson, J. C. Conrad and D. W. C. MacMillan, J. Am. Chem. Soc., 2011, 133, 1738; (b) Y. Lam and K. N. Houk, J. Am. Chem. Soc., 2014, 136, 9556.
10 X. Yang, R. J. Phipps and F. D. Toste, J. Am. Chem. Soc., 2014, 136, 5225.

11 (a) L.-S. Zheng, Y.-L. Wei, K.-Z. Jiang, Y. Deng, Z.-J. Zheng and L.-W. Xu, Adv. Synth. Catal., 2014, 356, 3769; (b) S. J. Kwon and D. Y. Kim, J. Fluorine Chem., 2015, 180, 201; (c) K. Hayamizu, N. Terayama, D. Hashizume, K. Dodo and M. Sodeoka, Tetrahedron, 2015, 71, 6594.

12 L. Zhang, N. Fu and S. Luo, Acc. Chem. Res., 2015, 48, 986.

13 The intramolecular F-attack with the secondary NH-F species (see ref. 9b), in situ transferred from the fluorination reagent to the aminocatalyst, though not completely excluded, may not be applicable in our case. First, there is no such precedence on fluorination with $\mathrm{NH}-\mathrm{F}$ species. In addition, even if an F-transfer did occur, facile proton release instead of F-attackmight be the dominant pathway, leading to an unreactive neutral-N-F species or even poisoning of the catalyst. Experimentally, we did not detect such species by in situ ESI-MS in both cases.

14 In TS-S, a short $\mathrm{C}-\mathrm{H} \cdots \mathrm{F}$ distance was found of $2.44 \AA$ A. Atoms in Molecules (AIM) analysis at the bond critical point suggested a weak interaction between $\mathrm{H}$ and $\mathrm{F}$ where the electron density $(\rho)$ is 0.0089 au and the Laplacian value $\left(\Delta \rho^{2}\right)$ is 0.037 au. These values indicate that a weak $\mathrm{C}-\mathrm{H} \cdots$ $\mathrm{F}$ interaction is present. For a review on $\mathrm{C}-\mathrm{H} \cdots \mathrm{F}-\mathrm{C}$ interaction, see: C.-C. Liu and M. C. W. Chan, Acc. Chem. Res., 2015, 48, 1580. 